\title{
Giardia duodenalis multi-locus genotypes in dogs with different levels of synanthropism and clinical signs
}

\author{
Mathilde Uiterwijk ${ }^{1,2}$, Lapo Mughini-Gras ${ }^{4,5}$, Rolf Nijsse ${ }^{1}$, Jaap A. Wagenaar ${ }^{1,3}$, Harm W. Ploeger ${ }^{1}$ \\ and Frans N. J. Kooyman ${ }^{1 *}$ (D)
}

\begin{abstract}
Background: In dogs, infections with Giardia duodenalis are mainly caused by assemblages $C$ and D, but also by the potentially zoonotic assemblages A and B. The aims of this study were to assess differences in assemblages (i) between dogs living mainly in close proximity to humans (synanthropic dogs) versus dogs living mainly among other dogs, (ii) between samples of dogs with or without loose stool, and (iii) related to the amount of cysts shedding.

Methods: One hundred eighty-nine qPCR Giardia positive fecal samples of dogs originating from four groups (household, sheltered, hunting, and dogs for which a veterinarian sent a fecal sample to a diagnostic laboratory) were used for genotyping. For this, multi-locus genotyping of beta-giardin, triose phosphate isomerase, and glutamate dehydrogenase and genotyping of SSU rDNA gene fragments were performed. Fecal consistency was scored (loose or non-loose stool), and cysts per gram of feces were determined with qPCR.

Results: Assemblage D was the most prevalent in all groups, followed by the other canid assemblage C. Also, mixed C/D was common. In two (synanthropic) household dogs, the potentially zoonotic assemblage Al was present. Although occurrence of assemblage Al in household dogs was not significantly different from dogs living among other dogs (sheltered and hunting dogs), it was significantly higher compared to dogs for which a sample was sent to a diagnostic laboratory. Dogs with assemblage $D$ shed significantly more cysts than dogs with other assemblages (except for mixed C/D results) or dogs in which no assemblage could be determined. None of the assemblages was significantly associated with loose stool.
\end{abstract}

Conclusion: Not only do dogs mainly shed the canid Giardia duodenalis assemblages D and/or C, the numbers of cysts per gram for the canid assemblage D were also higher than for the potential zoonotic assemblage Al. Based on the assemblages shed by dogs, the risk to public health posed by dogs is estimated to be low, even though the dogs that shed Al were synanthropic household dogs. Loose stool in infected dogs was not associated with any particular Giardia assemblage.

Keywords: Giardiosis, Assemblage, Canine, Zoonotic, Cysts per gram

*Correspondence: f.n.j.kooyman@uu.nl

${ }^{1}$ Department of Infectious Diseases and Immunology, Faculty of Veterinary Medicine, Utrecht University, Utrecht, The Netherlands

Full list of author information is available at the end of the article

\section{Background}

Giardia duodenalis (synonyms G. intestinalis and G. lamblia) is a common gastrointestinal parasite in humans and animals worldwide. This species can be further divided into eight assemblages denoted with letters A to $\mathrm{H}$, based upon substantial sequence differences $[1,2]$. 
Assemblages $\mathrm{C}$ to $\mathrm{H}$ are thought to have narrow host ranges, even though recent reports suggest that their host specificity might be broader than originally thought $[3,4]$.

Assemblages A and B are detected in humans in addition to several animal species, allowing for G. duodenalis to be considered a zoonotic agent [5]. Due to more recent insights into multi-locus genotypes (MLG), which further divide assemblages $A$ and $B$ in sub-assemblages with varying host ranges, the zoonotic importance of $G$. duodenalis is currently considered to be lower than previously thought [6-9].

Assemblages $C$ and D are canid-specific. However, other assemblages, particularly $\mathrm{A}$ and $\mathrm{B}$, can also be found in dogs $[10-13]$. The relative contribution of assemblage AI in synanthropic animals (defined as animals that live in close contact with humans), including dogs, can be as high as in humans themselves [6]. In contrast to this, other studies report predominantly canid assemblages in synanthropic dogs $[8,14,15]$. Use of multiple loci for detection of $G$. duodenalis has shown that mixed assemblage infections occur regularly in humans and many animals, including dogs [8, 16-19].

Although Giardia infections can cause gastrointestinal disease in dogs, there is no significant association of Giardia-positive fecal samples with diarrhea or loose stool [20-23]. However, the relation between fecal consistency and Giardia positivity has not been assessed as assemblage-specific. Also, the amount of cyst shedding has not been assessed assemblage-specifically, so it is not clear whether infections with canid assemblages $\mathrm{C}$ and $\mathrm{D}$ have a different host-pathogen interaction in dogs than infections with other assemblages, such as $\mathrm{A}$ and B. Results from an experimental study showed that dogs inoculated with trophozoites and cysts of human isolates developed no symptoms. However, dog numbers were low, and the isolates were not molecularly characterized [24].

Herewith, we aimed to determine whether synanthropic dogs are more likely to shed non-canid assemblages than dogs living among other dogs and whether infection with non-canid assemblages results in similar symptoms and numbers of cysts that are excreted. For this, G. duodenalis-positive dog fecal samples from four groups of dogs with different levels of synanthropism and differences in clinical signs were genotyped and the level of cyst shedding was determined.

\section{Methods}

\section{Dogs and diagnostic tests}

For this study, 189 G. duodenalis qPCR-positive dog samples obtained from previous studies were used. For more details about the study population, fecal sampling, DNA isolation, qPCR, rapid enzyme immunochromatographic assay (IDEXX SNAP ${ }^{\circledR}$ Giardia), direct immunofluorescence (DFA, Merifluor) and centrifugation sedimentation flotation (CSF) coproscopical analysis, we refer to those studies $[20,25]$. The household dogs $(n=47)$ were considered synanthropic dogs, whereas the sheltered $(n=48)$ and hunting dogs (scent hounds; $n=30$ ) were considered group-housed dogs, living predominantly among other dogs. The dogs for which a sample was sent to the Veterinary Microbiological Diagnostic Center (VMDC) of the Faculty of Veterinary Medicine of Utrecht University $(n=64)$ were left out of the synanthropic group, because detailed information about living conditions was not available for all dogs. This group will be further referred to as 'clinical dogs.' Fecal samples of the clinical dogs were submitted for parasite testing to diagnose a possible parasitic cause of clinical symptoms (mostly gastrointestinal), but also, to a lesser extent, for control of antiparasitic therapy or routine monitoring. Fecal samples were scored for consistency and classified as loose or nonloose as described [20].

\section{Multilocus genotyping}

MLG of gdh (glutamate dehydrogenase), bg (betagiardin), and tpi (triose phosphate isomerase) and genotyping of SSU rDNA (small subunit ribosomal DNA) gene loci was performed on the fecal samples by nested PCRs. See Additional file 1: Table S1 for primer sequences, amplicon size, and references. The nested PCRs on singlecopy loci were performed with DreamTaq DNA polymerase (Thermo Scientific) as described below, with several adjustments for optimization reasons. Nested PCR on $g d h$ was performed as described [26] with the following modifications: bovine serum albumin $(0.5 \mathrm{mg} / \mathrm{ml})$ was included in the PCR mixture, and the annealing temperatures were $57.5{ }^{\circ} \mathrm{C}$ and $60.0{ }^{\circ} \mathrm{C}$ for the first and second amplification, respectively. Nested PCR on $b g$ was performed as described [27] with an annealing temperature of $65.0{ }^{\circ} \mathrm{C}$ and $50.0{ }^{\circ} \mathrm{C}$ for the first and second amplifications, respectively. The first amplification of nested PCR on the tpi locus was performed as described [28], but with annealing temperature of $60.0^{\circ} \mathrm{C}$. The second amplification was assemblage-specific. Assemblage A-specific tpi amplification was achieved as described [19], but with an annealing temperature of $60.0^{\circ} \mathrm{C}$. Assemblage B-specific tpi amplification was achieved as described [29], but with an annealing temperature of $60.0{ }^{\circ} \mathrm{C}$. The nested PCR on the SSU-rDNA locus was performed with Phusion Hot Start II DNA Polymerase (Thermo Scientific) with the buffer for GC-rich templates and the inclusion of $8 \%$ DMSO. The primers were those described [30], and the annealing temperature was $65.0^{\circ} \mathrm{C}$. In all PCRs, positive and negative control templates were 
included. Templates from both assemblage A and B were included in both assemblage-specific PCRs. All amplicons were Sanger-sequenced at BaseClear (Leiden, The Netherlands). The sequences were aligned and a phylogenetic tree was constructed in DNASTAR Lasergene 14, together with reference sequences from all assemblages [6]. Based on the grouping with the reference sequences, the assemblage was determined.

\section{Nucleotide sequence accession numbers}

Giardia sequences generated in this study were deposited in the NCBI GenBank database under the following accession numbers: MW138896-MW138913 ( $g d h)$, MW138914-MW138934 (bg), and MW138935 (tpi).

\section{Statistical analyses}

Differences in occurrence of the different assemblages between dog groups were assessed using two-sample tests on the equality of proportions. The cysts per gram (CPG) were determined by qPCR with a calibration curve ranging from $3 \times 10^{6}$ to $300 \mathrm{CPG}$ [25]. The relationship between CPG and assemblages was assessed using negative binomial regression. A cluster-correlated robust variance estimator was included in the analyses to adjust for clustering of dogs at the household, shelter, or hunting dog group level, as described [20]. Statistical analysis was performed using STATA 16 (StataCorp LP, College Station, TX, USA).

\section{Results}

\section{Diagnostic tests and multilocus genotyping}

Of the 189 samples, 107 (56.6\%) samples yielded negative MLG or SSU rDNA genotyping results. Raw data for Giardia results including qPCR Cp, CPG, IDEXX SNAP $^{\circledR}$ Giardia, DFA, and CSF test for all samples are provided in Additional file 2: Table S2. CSF results of endoparasites in general are beyond the scope of this article and can be found in Uiterwijk et al. [20]. Table 1 shows detailed results of the 82 samples with $g d h, b g$, tpi, and/or SSU rDNA positive PCR results. Mixed C/D samples are defined as samples that showed double peaks in the sequences. This was shown with the loci SSU $r D N A(n=8)$ and $b g(n=1)$. Combined mixed C/D results are defined as samples containing assemblage $C$ based on one locus and assemblage $\mathrm{D}$ based on another locus, or mixed C/D. Of the 14 combined mixed C/Ds, 8 showed double peaks at the SSU rDNA locus at the position where assemblage $C$ and $D$ differ (see Additional file 3: Figure S1), and 6 showed assemblage $C$ with 1 locus and assemblage $\mathrm{D}$ with another, including $1 \mathrm{C} / \mathrm{D}$ at the $b g$ locus (Additional file 2: Table S2). The SSUrDNA sequences without a double peak are identical to the GenBank sequences GU126431 (assemblage AI),
Table 1 Distribution of the assemblages Al, C, mixed C/D, and D of MLG ( $g d h, b g$, and tpi loci) and SSU rDNA, and combined results

\begin{tabular}{lccccc}
\hline & Al & C & C/D & D & Total \\
\hline gdh & 1 & 6 & 0 & 11 & $18(9.5 \%)$ \\
bg & 1 & 6 & 1 & 13 & $21(11.1 \%)$ \\
tpi & 1 & 0 & 0 & 0 & $1(0.5 \%)$ \\
SSU rDNA & 2 & 17 & 8 & 55 & $82(43.4 \%)$ \\
Combined & 2 & 17 & 14 & 49 & 82 \\
\hline
\end{tabular}

\section{GU126436 (assemblage C), and GU126442 (assemblage} D).

In three dog groups, assemblage $\mathrm{D}$ was most prevalent. In the hunting dogs, both assemblage $\mathrm{D}$ and $\mathrm{C} / \mathrm{D}$ were highly present. Assemblage AI was only present in two household dogs (Table 2).

\section{Association of assemblage Al with synantropism}

When considering only the samples for which an assemblage was determined and adjusting for clustering, the difference in occurrence of assemblage AI between synanthropic dogs (household dogs; 18.2\%, 95\% CI $4.0-5.4 \%, n=11$ ) compared to dogs living among other dogs (sheltered and hunting dogs; $0.0 \%, 95 \%$ CI $0.0-10.0 \%, n=35)$ was not significant $(P=0.131)$. However, the occurrence of AI was significantly higher among household dogs compared to clinical dogs $(0.0 \%, 95 \%$ CI $0.0-9.7 \%, n=36$, two-sample test, $Z=-2.5813, P=0.010)$.

\section{Association of assemblages with loose stool}

Considering only the dogs with loose stool, there was no significant difference in the occurrence of AI between the dog groups. Indeed, none of the assemblage groups was significantly associated with loose stool. In the samples in which no assemblage could be determined, the CPG was significantly lower in the samples with loose stool $(4.6$ $\times 10^{3}, 95 \%$ CI $\left.2.7 \times 10^{3}-6.5 \times 10^{3}\right)$ than in the samples with no loose stool $\left(1.0 \times 10^{4}, 95 \%\right.$ CI $3.6 \times 10^{3}-1.7 \times$ $104, Z=-2.23, P=0.026)$.

\section{Association of assemblages with CPG}

The CPGs of the samples with assemblages $\mathrm{C}$, combined mixed $\mathrm{C} / \mathrm{D}$, or $\mathrm{D}$ (Table 2) were significantly higher than the CPG of the samples with undetermined assemblage $(P=0.000)$. CPGs of samples with assemblage D were significantly higher than the samples with assemblage C $(P=0.006)$, AI $(P=0.005)$, and the undetermined $(P=0.000)$ samples, but not with combined mixed C/D. 
Table 2 Occurrence (\%) and 95\% Cl (in brackets) of Giardia duodenalis assemblage Al, C, combined mixed C/D, D, and samples for which no assemblage could be determined, over the dog population

\begin{tabular}{|c|c|c|c|c|c|}
\hline Dog population & $\mathrm{Al}$ & C & $C / D$ & D & No assemblages determined \\
\hline $\begin{array}{l}\text { Household } \\
n=47\end{array}$ & $4.3(1.0-15.8)$ & $6.4(2.1-17.9)$ & 0 & $12.8(5.7-26.0)$ & $76.6(62.4-86.6)$ \\
\hline $\begin{array}{l}\text { Clinical } \\
n=64\end{array}$ & 0 & $15.6(8.6-26.6)$ & $3.1(0.8-11.9)$ & $32.8(22.5-45.1)$ & $48.4(36.2-60.9)$ \\
\hline $\begin{array}{l}\text { Sheltered } \\
n=48\end{array}$ & 0 & $6.3(1.4-24.0)$ & 0 & $22.9(13.5-36.1)$ & $70.8(57.5-81.4)$ \\
\hline $\begin{array}{l}\text { Hunting } \\
n=30\end{array}$ & 0 & $3.3(1.1-9.9)$ & $40.0(29.4-51.6)$ & $36.7(3.3-40.5)$ & $20.0(16.5-24.0)$ \\
\hline $\begin{array}{l}\text { Total } \\
n=189\end{array}$ & $1.1(0.3-4.3)$ & $9.0(5.4-14.6)$ & $7.4(2.6-19.4)$ & $25.9(20.2-32.6)$ & $56.6(45.6-67.0)$ \\
\hline $\begin{array}{l}\text { Mean CPG } \\
(95 \% \mathrm{Cl})\end{array}$ & $\begin{array}{l}3.3 \times 10^{4} \\
\left(1.1 \times 10^{3}-6.4 \times 10^{4}\right)\end{array}$ & $\begin{array}{l}9.3 \times 10^{4} \\
\left(2.2 \times 10^{4}-1.6 \times 10^{5}\right)\end{array}$ & $\begin{array}{l}6.5 \times 10^{4} \\
\left(1.0 \times 10^{4}-1.2 \times 10^{5}\right)\end{array}$ & $\begin{array}{l}4.7 \times 10^{5} \\
\left(4.3 \times 10^{4}-9.8 \times 10^{5}\right)\end{array}$ & $\begin{array}{l}9.1 \times 10^{3} \\
\left(3.9 \times 10^{3}-1.4 \times 10^{4}\right)\end{array}$ \\
\hline
\end{tabular}

Mean CPG and $95 \% \mathrm{Cl}$ (in brackets) are provided. Cl confidence interval

\section{Discussion}

To determine the (sub)assemblages of G. duodenalis shed by dogs and their association with synanthropism, clinical signs, and cyst shedding, we performed MLG and SSU rRNA PCR on qPCR-positive fecal samples from four groups of dogs. In $43.4 \%$ of the G. duodenalis qPCR-positive samples $(n=189)$, genetic characterization was successful. Due to the high sensitivity of the multicopy SSU rDNA, this locus yielded most positive results of the four loci. Canid assemblages $\mathrm{C}$ and $\mathrm{D}$ were the most prevalent in all dogs groups, which is in accordance with previous findings where dog-specific assemblages were most prevalent in household and sheltered dogs [14, 31-34]. The potential zoonotic assemblage AI is mainly found in pets and livestock, and to a lesser extent in humans [2]. Our findings are therefore also in accordance to reports in which AI was found in dogs [35], even though this assemblage was not most prevalent in our study. Assemblage AI was only detected in the synanthropic household dogs. Because spatiotemporal epidemiological and molecular information on humans and animals needs to be combined to demonstrate zoonotic transmission in a household [36, 37], the sources of AI in those two dogs remain unclear. The dogs may have contracted this genotype directly or indirectly from various sources, e.g. from humans, other dogs, or other animals. In turn, the dogs may infect other animals and people, even though only one dog was shown to have a patent infection (Additional File 2: Table S2). Molecular detection of an assemblage in stool could be due to a patent infection or merely mechanical passage. To detect patent infections, mRNA assays can be used [38] or cysts or cyst wall proteins can be detected in stool samples [25]. In our study, three assays to detect cysts or cyst wall proteins were performed; DFA, CSF, and IDEXX SNAP $^{\circledR}$ Giardia. In general, samples which tested positive with DFA, CSF, and/or IDEXX SNAP ${ }^{\circledR}$ Giardia showed higher CPG because of the lower sensitivities of these tests compared to qPCR [25]. This means that for samples with relatively lower CPGs, such as from the one AI dog, in many cases it could not be determined whether there was a true infection or merely passage.

The occurrence of assemblage AI was significantly higher among household dogs compared to the (predominantly synanthropic) clinical dogs. Both household dogs that shed AI did not have loose stool. But since none of the assemblage groups was significantly associated with loose stool, this does not indicate that dogs infected with assemblage AI may show less or more symptoms than with assemblages $C$ and/or D. We found significant differences in CPG shed for the different assemblages. Lower sensitivity of MLG and SSU rDNA nested PCRs compared to qPCR most likely accounts for the significant difference in CPG between MLG/SSU rDNA-negative and -positive samples. Assemblages D and combined mixed C/D showed the highest CPG compared to the other assemblages. This may indicate that infections with AI, C, C/D, and $\mathrm{D}$ behave differently in dogs with regard to cyst production. Possibly, canid-assemblages $\mathrm{C}$ and especially $\mathrm{D}$ are better adapted to dogs, thus leading to higher CPGs and relatively more often positive MLG and SSU $r D N A$ results. This might have led to an underreporting of samples with sub-assemblage such as AI. Also, this may contribute to the relatively more reports of canid-assemblages in dogs compared to non-canid assemblages mentioned above [6, 14, 31-35]. Use of Next Generation Amplicon Sequencing might resolve the problem of underreporting of certain assemblages, 
because with this sequencing method, assemblages present in mixed infection can be separately detected. Also, infections with lower CPG can be detected [38].

The finding of lower CPG in samples with loose stool for which no assemblage could be determined may be explained by the fact that nested PCRs showed higher sensitivity on purified cysts [25] and possibly also on diluted feces (e.g. loose stool). In our study, no assemblage B or other subtypes of assemblage A such as AII were detected, which have been found in dogs before [12, 39, 40]. Traub et al. (2009) defined three transmission cycles of Giardia assemblages in dogs: anthroponotic, zoonotic, and dog-specific [41]. Which assemblage prevails in dogs seems to depend on factors related to dogs, such as a high level of coprophagic behavior [42], the level of contact with humans and living conditions, factors related to the parasite, such as host-specific adaptations of assemblages, and factors related to the human population, such as Giardia prevalence and circulating assemblages [43]. Once an assemblage is introduced, it may circulate relatively easily among (groups of) dogs. For example, the high prevalence of $\mathrm{D}$ and $\mathrm{C} / \mathrm{D}$ in the hunting dogs in our study can be explained by living conditions and behavior favorable for feco-oral transmission, introduction of dogspecific assemblages due to more contact with canids than with humans, and the relatively low prevalence of Giardia in the Dutch human population [44, 45]. Also, this may explain, at least partly, the reports of high versus low risks of zoonotic transmission [7, 39, 46, 47].

\section{Conclusions}

Giardia infections in both synanthropic and non-synanthropic dogs were mainly caused by canid assemblages, with the potentially zoonotic assemblage AI in just two synanthropic dogs (1.1\% of all dogs). Dogs with canid assemblages, especially assemblage $D$, showed much higher CPGs. This may suggest that the assemblage AI, although capable of infecting dogs, has a different hostpathogen interaction and is possibly less able to multiply in dogs compared to the canid assemblages. Based upon our results, the zoonotic risk of Giardia infections in dogs is low.

\section{Supplementary information}

Supplementary information accompanies this paper at https://doi. org/10.1186/s13071-020-04496-2.

Additional file 1: Table S1. Characteristics of multi-locus genotype nested PCRs.

Additional file 2: Table S2. Raw data.

Additional file 3: Figure S1. Example of SSU-rDNA fragment with "double peaks" in the Sanger sequence results.

\section{Abbreviations}

bg: Beta-giardin; Cl: Confidence interval; CPG: Cyst per gram feces; gdh: Glutamate dehydrogenase; MLG: Multi-locus genotyping; $P$ : $P$-value; qPCR: Quantitative real-time PCR; tpi: Triose phosphate isomerase; SSU rDNA: Small subunit ribosomal DNA; VMDC: Veterinary Microbiological Diagnostic Center.

\section{Acknowledgements}

The Veterinary Microbiological Diagnostic Centre and the Shelter Medicine Programme at the Department of Clinical Sciences of Companion Animals, both of the Faculty of Veterinary Medicine of Utrecht University, and the students in training performing their research internship are acknowledged for their substantial contributions and input. The owners of the dogs and staff of the kennels are acknowledged for their cooperation.

\section{Authors' contributions}

MU, FNJK, JAW, RN, LMG and HWP conceived and designed the study. MU, FNJK, and RN were involved in collection and processing of the samples. FNJK conducted the optimizations and experiments. JAW and HWP were project leaders and made conceptual contributions. LMG performed the statistical analysis, in consultation with MU and FNJK. MU and FNJK wrote the manuscript. All authors provided relevant input at different stages of manuscript preparation. All authors read and approved the final manuscript.

\section{Funding}

Not applicable.

\section{Availability of data and materials}

The datasets used and/or analysed during the present study are presented in the article and its additional files, or are available from the corresponding author upon reasonable request.

\section{Ethics approval and consent to participate}

Not applicable.

\section{Consent for publication}

Not applicable.

\section{Competing interests}

The authors declare that they have no competing interests.

\section{Author details}

${ }^{1}$ Department of Infectious Diseases and Immunology, Faculty of Veterinary Medicine, Utrecht University, Utrecht, The Netherlands. ${ }^{2}$ Present Address: National Reference Laboratory, The Netherlands Food and Consumer Product Safety Authority (NVWA), Centre for Monitoring of Vectors (CMV), Wageningen, The Netherlands. ${ }^{3}$ Wageningen Bioveterinary Research, Lelystad, The Netherlands. ${ }^{4}$ National Institute for Public Health and the Environment (RIVM), Centre for Infectious Disease Control (Clb), Bilthoven, The Netherlands. ${ }^{5}$ Institute for Risk Assessment Sciences (IRAS), Faculty of Veterinary Medicine, Utrecht University, Utrecht, The Netherlands.

Received: 2 September 2020 Accepted: 19 November 2020 Published online: 02 December 2020

References

1. Plutzer J, Ongerth J, Karanis P. Giardia taxonomy, phylogeny and epidemiology: facts and open questions. Int J Hyg Envir Heal. 2010;213:321-33.

2. Ryan U, Cacciò SM. Zoonotic potential of Giardia. Int J Parasitol. 2013:43:943-56.

3. Caccio SM, Lalle M, Svard SG. Host specificity in the Giardia duodenalis species complex. Infect Genet Evol. 2017;66:335-45.

4. Štrkolcová G, Madár M, Hinney B, Goldová M, Mojžišová J, Halánová M. Dog's genotype of Giardia duodenalis in human: first evidence in Europe. Acta Parasitol. 2015;60:796-9.

5. van Keulen H, Macechko PT, Wade S, Schaaf S, Wallis PM, Erlandsen SL. Presence of human Giardia in domestic, farm and wild animals, and environmental samples suggests a zoonotic potential for giardiasis. Vet Par. 2002;108:97-107. 
6. Sprong H, Caccio SM, van der Giessen JW. Identification of zoonotic genotypes of Giardia duodenalis. PLoS Negl Trop Dis. 2009;124:107-12.

7. Lebbad M, Petersson I, Karlsson L, Botero-Kleiven S, Andersson JO, Svenungsson B, et al. Multilocus genotyping of human Giardia isolates suggests limited zoonotic transmission and association between assemblage $B$ and flatulence in children. PLoS Negl Trop Dis. 2011;5.

8. Beck R, Sprong H, Pozio E, Cacciò SM. Genotyping Giardia duodenalis isolates from dogs: lessons from a multilocus sequence typing study. Vector Borne Zoonotic Dis. 2012:12:206-13.

9. de Lucio A, Bailo B, Aguilera M, Cardona GA, Fernández-Crespo JC, Carmena D. No molecular epidemiological evidence supporting household transmission of zoonotic Giardia duodenalis and Cryptosporidium spp. from pet dogs and cats in the province of Álava, Northern Spain. Acta Trop. 2017:170:48-56.

10. Covacin C, Aucoin DP, Elliot A, Thompson RCA. Genotypic characterisation of Giardia from domestic dogs in the USA. Vet Par. 2011;177:28-32.

11. Claerebout E, Casaert S, Dalemans AC, De Wilde N, Levecke B, Vercruysse J, et al. Giardia and other intestinal parasites in different dog populations in Northern Belgium. Vet Par. 2009;161:41-6.

12. Gil H, Cano L, de Lucio A, Bailo B, de Mingo MH, Cardona GA, et al. Detection and molecular diversity of Giardia duodenalis and Cryptosporidium spp. in sheltered dogs and cats in Northern Spain. Infect Genet Evol 2017. 50:62-9.

13. Fantinatti M, Caseca AC, Bello AR, Fernandes O, Da-Cruz AM. The presence of Giardia lamblia assemblage A in dogs suggests an anthropozoonotic cycle of the parasite in Rio de Janeiro Brazil. Infect Genet Evol. 2018:65:265-9.

14. Kim HY, Lee H, Lee SH, Seo MG, Yi S, Kim JW, et al. Multilocus genotyping and risk factor analysis of Giardia duodenalis in dogs in Korea. Acta Trop. 2019:199:105113.

15. Cooper MA, Sterling CR, Gilman RH, Cama V, Ortega Y, Adam RD. Molecular analysis of household transmission of Giardia lamblia in a region of high endemicity in Peru. J linfec Dis. 2010;202:1713-21.

16. Almeida A, Pozio E, Cacciò SM. Genotyping of Giardia duodenalis cysts by new real-time PCR assays for detection of mixed infections in human samples. Appl Environ Microbiol. 2010;76:1895-901.

17. Adell-Aledón M, Köster PC, de Lucio A, Puente P, Hernández-de-Mingo $M$, Sánchez-Thevenet $P$, et al. Occurrence and molecular epidemiology of Giardia duodenalis infection in dog populations in eastern Spain. BMC Vet Res. 2018;14:26.

18. Rehbein S, Klotz C, Ignatius R, Müller E, Aebischer A, Kohn B. Giardia duodenalis in small animals and their owners in Germany: a pilot study. Zoonoses Public Health. 2019;66:117-24.

19. Geurden T, Geldhof P, Levecke B, Martens C, Berkvens D, Casaert S, et al. Mixed Giardia duodenalis assemblage A and E infections in calves. Int J Parasitol. 2008;38:259-64.

20. Uiterwijk M, Nijsse R, Kooyman FNJ, Wagenaar JA, Mughini-Gras L, Ploeger HW. Host factors associated with Giardia duodenalis infection in dogs across multiple diagnostic tests. Parasit Vectors. 2019;12:556-65.

21. Bugg RJ, Robertson ID, Elliot AD, Thompson RCA. Gastrointestinal parasites of urban dogs in Perth Western Australia. Vet J. 1999;157:295-301.

22. Gizzi ABDR, Oliveira ST, Leutenegger CM, Estrada M, Kozemjakin DA, Stedile $R$, et al. Presence of infectious agents and co-infections in diarrheic dogs determined with a real-time polymerase chain reaction-based panel. BMC Vet Res. 2014;10

23. Upjohn M, Cobb C, Monger J, Geurden T, Claerebout E, Fox M. Prevalence, molecular typing and risk factor analysis for Giardia duodenalis infections in dogs in a central London rescue shelter. Vet Par. 2010;172:341-6.

24. Rosa LAG, Gomes MA, Mundim AV, Mundim MJS, Pozzer EL, Faria ESM, et al. Infection of dogs by experimental inoculation with human isolates of Giardia duodenalis: clinical and laboratory manifestations. Vet Par. 2007;145:37-44

25. Uiterwijk M, Nijsse R, Kooyman FNJ, Wagenaar JA, Mughini-Gras L, Koop G, et al. Comparing four diagnostic tests for Giardia duodenalis in dogs using latent class analysis. Parasit Vectors. 2018;439-46.

26. Cacciò SM, Beck R, Lalle M, Marinculic A, Pozio E. Multilocus genotyping of Giardia duodenalis reveals striking differences between assemblages A and B. Int J Parasitol. 2008;38:1523-31.

27. Tseng YC, Ho GD, Chen $T$, Huang BF, Cheng PC, Chen JL, et al. Prevalence and genotype of Giardia duodenalis from faecal samples of stray dogs in Hualien city of eastern Taiwan. Trop Biomed. 2014;31:305-11.

28. Sulaiman IM, Fayer R, Bern C, Gilman RH, Trout JM, Schantz PM, et al. Triosephosphate isomerase gene characterization and potential zoonotic transmission of Giardia duodenalis. Emerg Infect Dis. 2003;9:1444-52.
29. Levecke B, Geldhof P, Claerebout E, Dorny P, Vercammen F, Cacciò SM, et al. Molecular characterisation of Giardia duodenalis in captive non-human primates reveals mixed assemblage $A$ and $B$ infections and novel polymorphisms. Int J Parasitol. 2009;39:1595-601.

30. Cacciò SM, Beck R, Almeida A, Bajer A, Pozio E. Identification of Giardia species and Giardia duodenalis assemblages by sequence analysis of the $5.8 \mathrm{~S}$ rDNA gene and internal transcribed spacers. Parasitology. 2010;137:919-25.

31. Qi M, Dong H, Wang R, Li J, Zhao J, Zhang L, et al. Infection rate and genetic diversity of Giardia duodenalis in pet and stray dogs in Henan Province China. Parasitol Int. 2016;65:159-62.

32. Pallant $L$, Barutzki D, Schaper R, Thompson RCA. The epidemiology of infections with Giardia species and genotypes in well cared for dogs and cats in Germany. Parasites Vectors. 2015;8.

33. Overgaauw PAM, van Zutphen L, Hoek D, Yaya FO, Roelfsema J, Pinelli E, et al. Zoonotic parasites in fecal samples and fur from dogs and cats in The Netherlands. Vet Par. 2009;163(1-2):115-22.

34. Tangtrongsup S, Scorza AV, Reif JS, Ballweber LR, Lappin MR, Salman MD. Seasonal distributions and other risk factors for Giardia duodenalis and Cryptosporidium spp. infections in dogs and cats in Chiang Mai, Thailand. Prev Vet Med. 2020;174:104820.

35. Leonhard S, Pfister K, Beelitz P, Wielinga C, Thompson RC. The molecular characterisation of Giardia from dogs in southern Germany. Vet Par. 2007;150:33-8.

36. Paulos $\mathrm{S}$, Köster $\mathrm{PC}$, de Lucio A, Hernández-de-Mingo M, Cardona GA, Fernández-Crespo JC, et al. Occurrence and subtype distribution of Blastocystis sp. in humans, dogs and cats sharing household in northern Spain and assessment of zoonotic transmission risk. Zoonoses Public Health. 2018;65:993-1002.

37. Ballweber LR, Xiao L, Bowman DD, Kahn G, Cama VA. Giardiasis in dogs and cats: update on epidemiology and public health significance. Trends Parasitol. 2010;26:180-9.

38. Thompson RCA, Ash A. Molecular epidemiology of Giardia and Cryptosporidium infections - What's new? Infect Genet Evol. 2019;75:103951.

39. Inpankaew T, Schär F, Odermatt P, Dalsgaard A, Chimnoi W, Khieu V, et al. Low risk for transmission of zoonotic Giardia duodenalis from dogs to humans in rural Cambodia. Parasites Vectors. 2014;7.

40. Quadros RM, Weiss PH, Marques SM, Miletti LC. Potential cross-contamination of similar Giardia duodenalis assemblages in children and pet dogs in Southern Brazil, as determined by, PCR-RFLP. Rev Inst Med Trop Sao Paulo. 2016;58:66.

41. Traub RJ, Inpankaew T, Reid SA, Sutthikornchai C, Sukthana Y, Robertson ID, et al. Transmission cycles of Giardia duodenalis in dogs and humans in Temple communities in Bangkok-A critical evaluation of its prevalence using three diagnostic tests in the field in the absence of a gold standard. Acta Trop. 2009;111:125-32.

42. Nijsse R, Mughini-Gras L, Wagenaar JA, Ploeger HW. Coprophagy in dogs interferes in the diagnosis of parasitic infections by faecal examination. Vet Par. 2014;204:304-9.

43. Palmer CS, Traub RJ, Robertson ID, Devlin G, Rees R, Thompson RCA. Determining the zoonotic significance of Giardia and Cryptosporidium in Australian dogs and cats. Vet Par. 2008;154:142-7.

44. Leung AKC, Leung AAM, Wong AHC, Sergi CM, Kam JKM. Giardiasis: an Overview. Recent Pat Inflamm Allergy Drug Discov. 2019;13:134-43.

45. ten Hove R, Schuurman T, Kooistra M, Möller L, van Lieshout L, Verweij JJ. Detection of diarrhoea-causing protozoa in general practice patients in The Netherlands by multiplex real-time PCR. Clin Microbiol Inf. 2007;13:1001-7.

46. Lee MF, Cadogan P, Eytle S, Copeland S, Walochnik J, Lindo JF. Molecular epidemiology and multilocus sequence analysis of potentially zoonotic Giardia spp. from humans and dogs in Jamaica. Parasitol Res. 2017;1 16:409-14.

47. Dado D, Montoya A, Blanco MA, Miro G, Saugar JM, Bailo B, et al. Prevalence and genotypes of Giardia duodenalis from dogs in Spain: possible zoonotic transmission and public health importance. Parasitol Res. 2012;111:2419-22.

\section{Publisher's Note}

Springer Nature remains neutral with regard to jurisdictional claims in published maps and institutional affiliations. 